\title{
The Origins of the Second Wave of COVID-19 in India - a Model-based \\ Investigation
}

\section{B SHAYAK ${ }^{(*)}$}

Theoretical and Applied Mechanics, Mechanical and Aerospace Engg, Cornell University,

Ithaca - 14853,

New York State, USA
MOHIT M SHARMA

Population Health Sciences, Weill Cornell Medicine, 1300 York Avenue, NYC - 10065,

New York State, USA

(*) Corresponding author. Email : sb2344@,cornell.edu, shayak.2015@iitkalumni.org

ORCID : 0000-0003-2502-2268

Tuesday 08 June 2021

---- 0 ----

Keywords. Large scale gatherings Mutant strain Small scale gatherings Unknown dynamical phenomena

Word counts. Abstract - 350, Text only - 8672, Grand total - 10680

$$
\text { ---- } 0 \text {---- } 0 \text {---- } 0 \text {---- } \quad \text {---- } 0 \text {---- } 0 \text {---- } 0 \text {---- }
$$




\begin{abstract}
Background. After months of declining or steady rates of daily COVID-19 cases, in April 2021 India experienced the onset of a massive second wave of the epidemic which overstressed its healthcare facilities and caused lakhs of casualties. During the wave, the reproduction number $R$ in some places was 1.8 or greater. The origins of this wave are at best partially understood. Socioeconomic activities had been running at a relatively high level for several months prior to the wave, and there was no relaxation of nonpharmaceutical interventions (NPI) in the immediately preceding days. A causal connection between the second wave and organized gatherings such as election activities, religious festivals and farmers' protests is impossible to establish due to absence of spatio-temporal correlation. Analysis of genomic sequencing data suggests that the emergence of the double mutant B1.617 and its sub-lineages cannot be the sole cause of the wave as well.

Methods and results. In a companion study [1] we have constructed a mathematical model for COVID-19 transmission which accounts for heterogeneity in people's interaction patterns. This model yields two phenomena of concern which are outside the purview of classical infectious disease dynamical understanding. These phenomena are $\bullet$ cryptogenic instability in which relatively small-scale social gatherings such as marriage functions and birthday parties play a disproportionately high role in driving spread, and - critical mass effect in which very low case counts result in a temporary and artificial suppression of an existing instability.

Principal findings. We believe that the above two phenomena are primarily responsible for the suddenness and severity of the second wave of COVID-19 in India. Complacency prevailing in early 2021 and emergence of mutant strain are not root causes of the wave but contributory factors to disease burden. The origins of the wave are complex and multifactorial, being largely governed by infectious disease dynamics which were unknown at the time of its onset. The lessons learned from the experience must be used to guide future course of NPI, not just in India but in other countries as well until significant fractions of their respective populations have been vaccinated.
\end{abstract}




\section{INTRODUCTION}

\$1. India : a case history. The first COVID-19 case was detected in India on 30 January 2020, the patient being a medical student returning from Wuhan, China. Despite some border controls (thermal screening and travel history declaration) being setup quite early, community transmission was initiated and by March, exponential case growth was observed. A full national lockdown was imposed starting 25 March; while it failed to have the intended effect of eliminating the disease, it did retard the spreading rate and allowed time for medical infrastructure to be developed and/or improved in the event of a wave. With effect from 08 June, a series of unlocking measures was instituted to maximize economic recovery. The epidemic grew slowly but monotonically, reaching a maximum of 97,860 new daily cases on 16 September, after which the rate began to fall. (All India data are taken from the tracking website [2]; data reported by other sites can show some differences but they are extremely minor.)

The unlocking procedure, which was partially paused but not reversed during the peak, continued after the cases began declining until by November 2020, restaurants, bars, cinema halls and other entertainment venues were all open. Wedding functions of upto 50 people and funeral gatherings of 20 were also permitted, as were gatherings of upto size 100 in places of worship. Metro and local train services in cities like Kolkata, Delhi and Mumbai were cautiously given the green light, while Indian Railways steadily increased the number of long-distance services. As domestic travel quarantine requirements eased, airlines started inching back towards their 2019 traffic levels. Mask mandates remained in force everywhere. During the reopening, in October, a new strain of the virus called B1.617 was detected in Maharashtra [3]. This strain is colloquially referred to as the "double mutant" since two of its fifteen mutations relative to the wildtype are potentially troublesome - one at position 452 is possibly associated with increased transmissibility and another at position 484 (position 478 in the sub-lineage B1.617.2) might be linked to immune escape. Despite the emergence of this strain and the gradual reopening measures, infections decreased steadily and on the last day of 2020, India recorded just above 19,000 COVID-19 cases. The case trajectories in India from the date of the index case upto New Year's Eve are shown below. The cumulative caseload is shown as a blue line associated with the right hand $y$-axis while the daily case rate is shown as grey bars associated with the left hand $y$-axis.

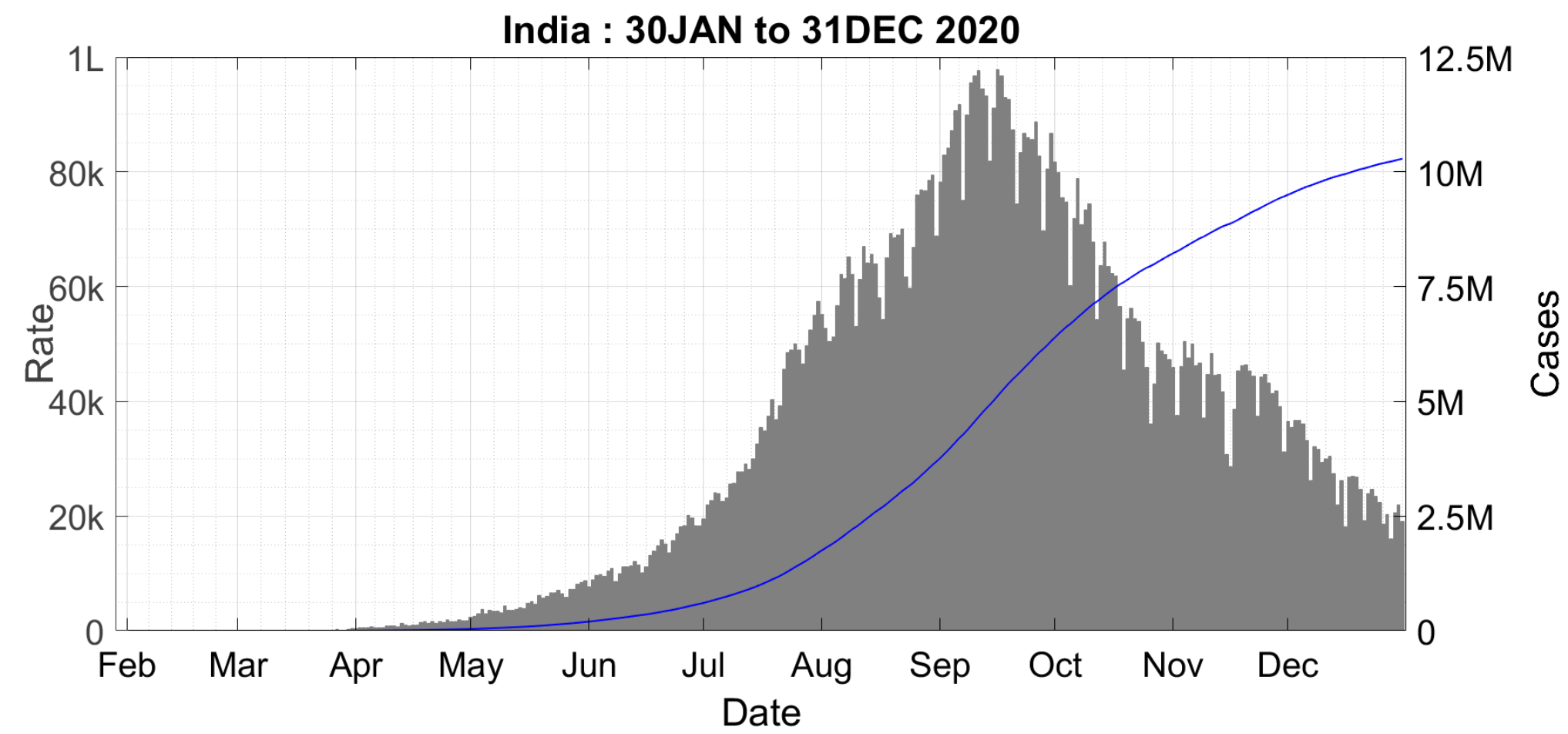

Figure 1 : Case trajectories in India from the date of the first discovered case to the end of 2020. The symbol ' $k$ ' denotes thousand, 'L' lakh and 'M' million. Labels on the x-axis correspond to the first day of each month.

During the final quarter of 2020, the test positivity rate also declined steadily, suggesting that the reduction in cases was not just an artefact of reduced testing rates. This is not to say that post- 15 September, cases decreased monotonically everywhere in India. Localized mini-waves corresponding to festival season were 
seen in Delhi, Kolkata, Mumbai as well as other places. In each instance, renewed emphasis on nonpharmaceutical interventions (NPI) served to bring the outbreak under control well before it reached the level of a public health crisis. Below we show these mini-waves in two regions, West Bengal and Delhi.
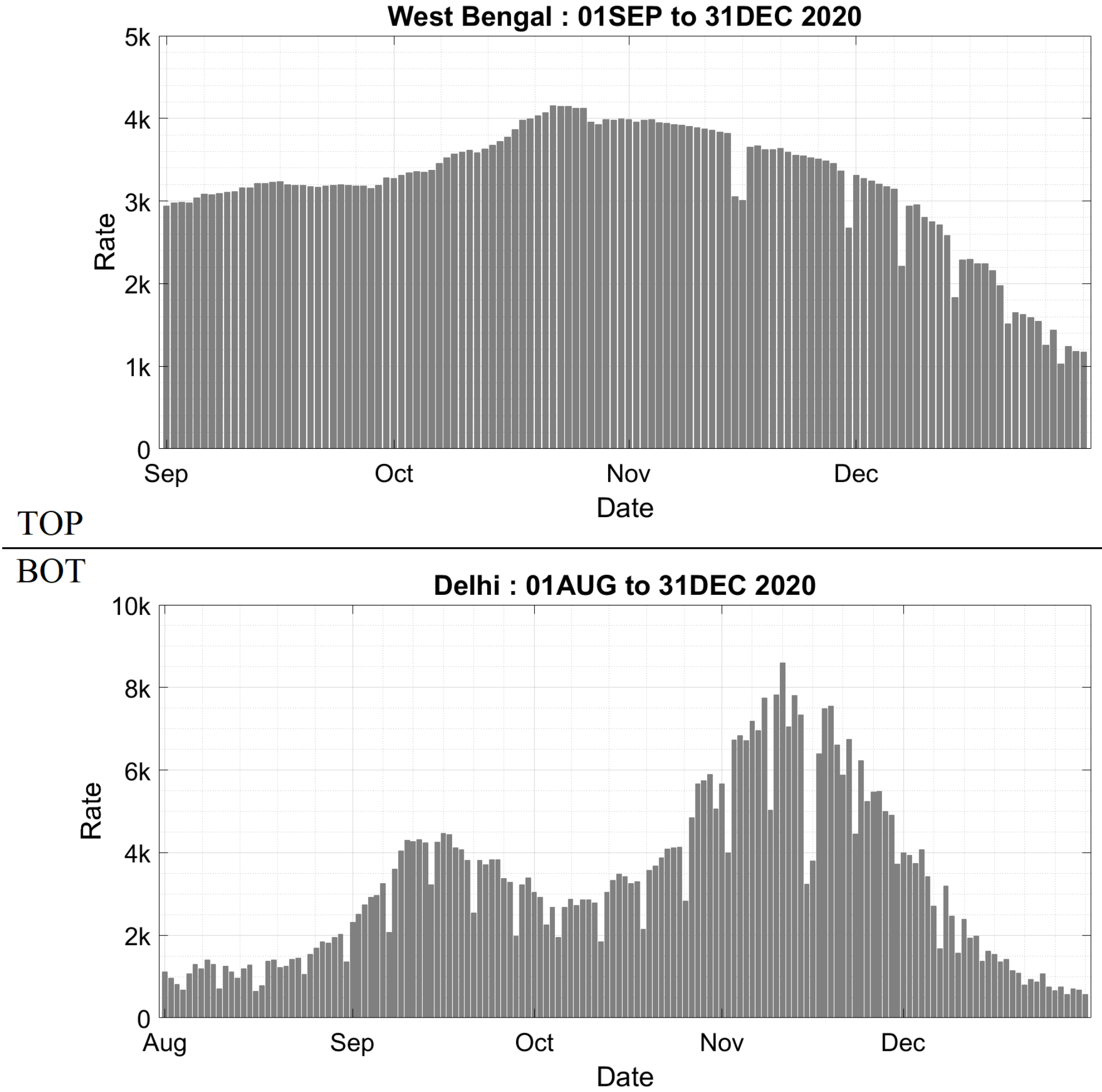

Figure 2 : Case trajectories in West Bengal and Delhi during the periods shown in the panel titles. The symbol ' $k$ ' denotes thousand. Labels on the x-axis correspond to the first day of each month. Durga Puja was held in West Bengal from 22 to 26 October; the (very flat) peak around this time probably corresponds to an increase in shopping and related activities in the days immediately preceding. Dushehra was on 26 October and Diwali on 14 November, right around the time of the second peak in Delhi.

Sixteen days into the new year, India reached another milestone in its fight against COVID-19. On this day, the vaccination drive was initiated using two vaccines - the indigenous candidate Covaxin developed by the Indian Council for Medical Research (ICMR) together with Bharat Biotech, and the vaccine Covishield, which is the Oxford/ Astra Zeneca candidate manufactured locally by Serum Institute of India. "Medicine and discipline" ("दवाई भी कड़ाई भी" in the Hindi original) was the slogan adopted to emphasize the need for continued adherence to NPI but enforcement became increasingly lax. The gathering size permitted at weddings was raised from 50 to 100 or even 200 in some places. On 18 January, India recorded less than ten thousand cases for the first time since the beginning of the pandemic. After an encouraging fortnight, the expert panel [4] agreed with everyone else (ourselves included) that the probability of a second peak was very low [5]. In the background of more four-figure days in February, the Central Government 
decided to permit full-scale multi-phase State Legislative Assembly elections in West Bengal, Assam, Tamil $\mathrm{Nadu}$, Kerala and Puducherry during the period March-May. The massive religious/ cultural festival of Kumbh Mela, scheduled to be held in Haridwar (Uttarakhand) during February to April, was also given a go-ahead albeit with a curtailed schedule (April only [6]) and a (nominal) mask mandate in place. Collective protests and dharnas by farmers against certain government legislation also continued unabated in the states of Punjab and Haryana and to a lesser extent in Delhi. The case trajectories for India during this quiescent phase are shown in the Figure below.

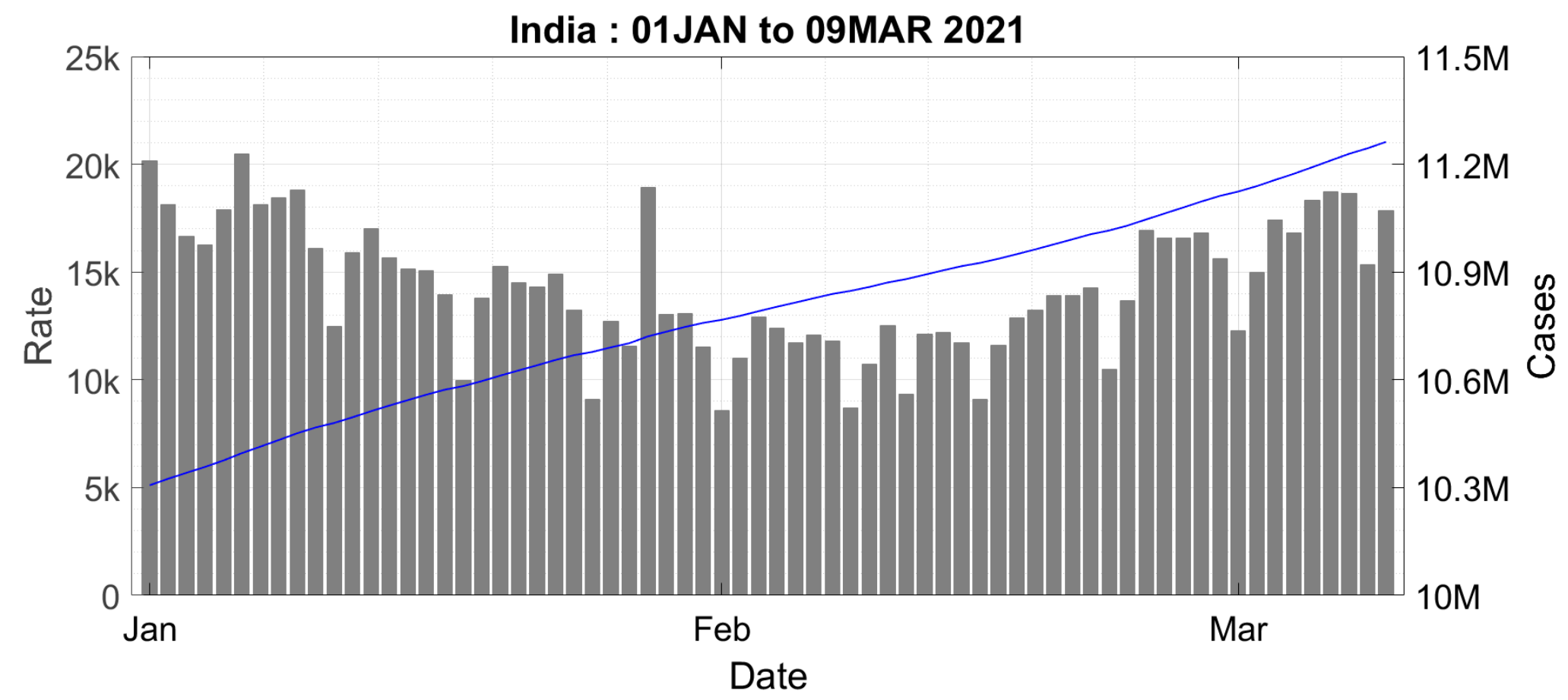

Figure 3 : Case trajectories in India in early 2021. The symbol ' $k$ ' denotes thousand and 'M' million. Labels on the xaxis correspond to the first day of each month.

On 01 March, all Indian citizens over 60 years of age were allowed access to the coronavirus vaccines. Six days later, the Ministry of Health and Family Welfare (MoHFW) expressed confidence that India was in the endgame phase of the pandemic and urged citizens to receive the vaccines when their turn came [7]. So low was the local demand for vaccines that six crores of doses were exported to almost 90 countries while COVID-19 immunization clinics in India saw doses lying on the shelves. The extent of the complacency can be imagined from the fact that despite the behavioural guidelines remaining in force, even eminent personalities made maskless public appearances.

In early March however, case counts started going up in Maharashtra. The level of about 3000-4000 daily cases which had been maintained all February became 10,000-plus on 06 March. These fluctuations appeared very similar to the mini-waves of Fig. 2 and nobody was particularly concerned. Fifteen days later however, the state registered 30,500 cases, which was for it an all-time maxima. Only as the rate kept climbing relentlessly, the state authorities became aware of a serious threat and started imposing progressively increasing social restrictions, but by then the damage had already been done. A peak case rate of 69,000 was recorded on 18 April before the statewide curfew succeeded in halting the rise. Shortly after Maharashtra, things started deteriorating in Delhi. Unlike in Maharashtra, the rise here was meteoric. From less than 400 daily cases on 10 March, the national capital went up to 800 on 20 March, then to 2800 on 01 April, 8000 on 10 April and a record peak of 28,000 on 20 April. A full lockdown was declared two or three days before the second peak but healthcare systems had already been strained beyond their limits. The third state to explode like a bomb was Uttar Pradesh. From less than 500 cases upto 20 March, there were 2600 on 01 April and 13,000 on 10 April. The peak of 38,000 was attained on 24 April with the entire state under evening curfew and weekend lockdown. In Fig. 4 we present the time traces of these three states when the wave hit. 

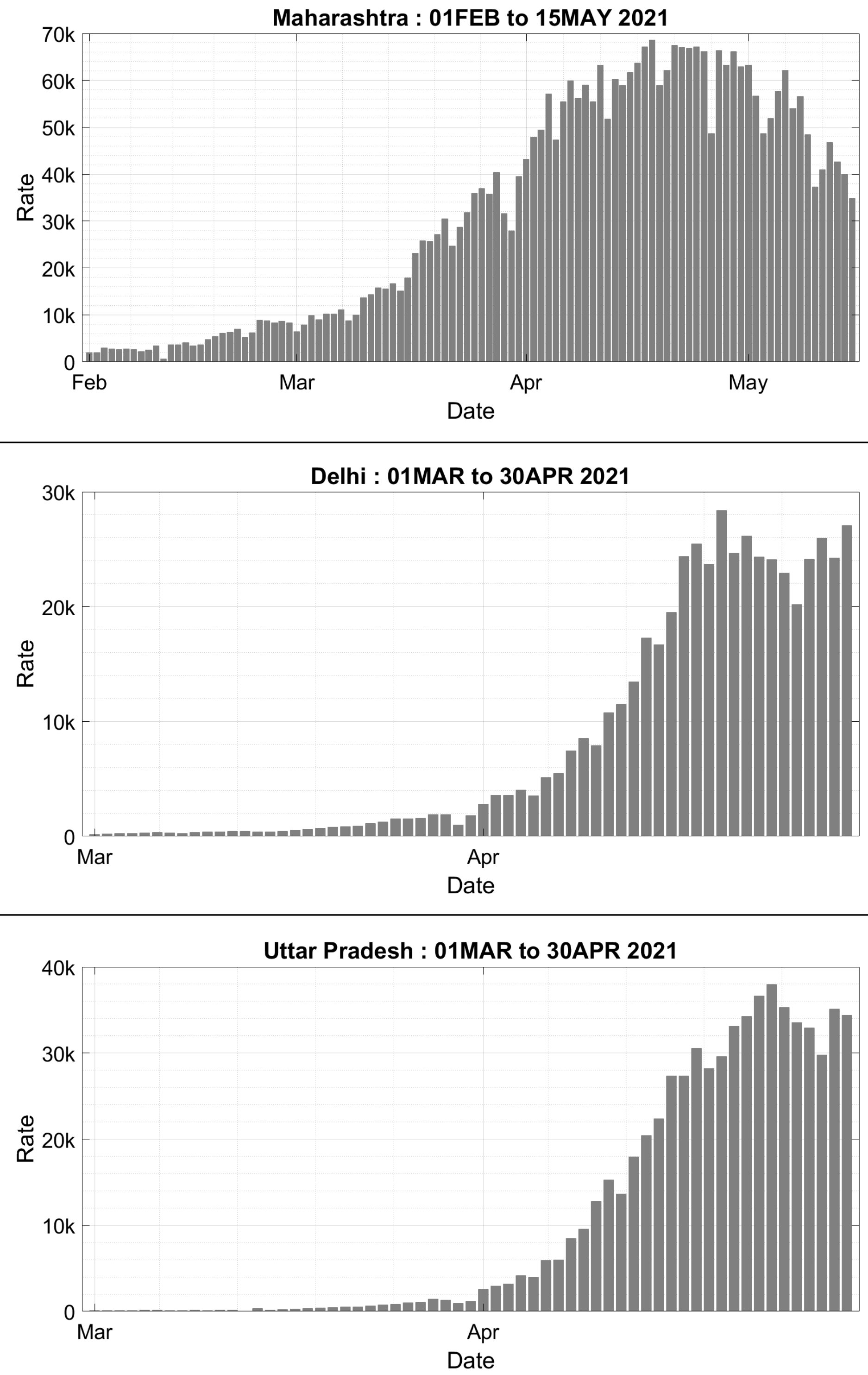

Figure 4 : Case trajectories in the hotspot states during the second wave. The symbol ' $k$ ' denotes thousand. Labels on the $\mathrm{x}$-axis correspond to the first day of each month. In Maharashtra, the wave begins from a moderate baseline and climbs comparatively gradually while in Delhi and Uttar Pradesh, the baseline is nearly zero and the rate of ascent blinding. Compare the middle panel here with Fig. 2-bot for an indication of what Delhi was prepared for and what it got instead. 
During the second wave, similar though less dramatic increases were seen all across the country - in the remaining states, either the absolute numbers were lower or the rates more gradual (for example Karnataka reached a maxima of 50,000 daily cases but the rate grew only tenfold in a month). The national peak of 4,14,280 cases was attained on 06 May, with that number being the highest of any country in the world. Unlike during the first peak when healthcare facilities by and large operated smoothly, the abrupt arrival of the second wave caught medical systems off guard with tragic consequences. Avoidable deaths, which were very few in number during the first wave, became rife during the second. There were accidents in two Maharashtra hospitals, both causing huge loss of life. Many hospitals in Delhi and Uttar Pradesh ran out of medical oxygen as the demand outstripped the supply by multiple times; patients who were attached to oxygen machines lost their lives. Hospital beds in the hotspot regions were filled to capacity and patients had to be turned away or kept waiting outside in ambulances; many of these died as well. Deaths due to accidents in healthcare settings and unavailability of oxygen and medical resources occurred in other states too such as Karnataka, Andhra Pradesh and Goa, although there they were thankfully fewer in number. The vaccines which only a month ago appeared so plentiful now became scarce as lakhs of people jostled each other for the life-saving shots; many arrived at vaccine clinics early in the morning only to go home empty-handed at the end of the day.

Amid the heart-rending stories, there were also uplifting ones. A philanthropic small businessman in Uttar Pradesh whose factory happened to use oxygen worked round the clock fetching empty cylinders from hospitals in his car, filling the cylinders and returning them, all for free. A Bollywood actor used his influence to access oxygen and drugs like faviparivir, and his private jet to airlift COVID-19 patients from cities without hospital beds to cities with beds. Catching the disease in the process, he fortunately recovered following mild symptoms. On a larger scale, a notable petrochemical corporation has been diverting oxygen from its oil refineries to states in need, free of cost. Indian Railways has been running Oxygen Express trains between states with high requirement (such as Maharashtra) and steel plants in Jharkhand, Odisha and West Bengal where industrial oxygen is available in bulk. These roll-on-roll-off (RORO) trains consist of flat goods wagons each carrying a tanker lorry. The empty tankers roll onto the train at the requirement state, the train travels to the steel plant where the tankers fill up and the train then returns to its source, where the tankers roll off and rush to hospitals. This way, one train can serve multiple cities in the same state with maximum efficiency and speed. The Government of India has sanctioned grants of nearly 5000 crore rupees to Bharat Biotech and Serum Institute of India to accelerate the production of Covaxin and Covishield. The international community has also been active in helping India mitigate the fallout of the disaster; hundreds of oxygen machines have been flown from New York City while the US has also promised supplies from its stockpile of the Oxford/ Astra Zeneca vaccine.

As we write this, the worst of the second wave is behind us. On 09 May, India recorded a reduction in active cases for the first time since early March. Active cases further decreased every day until now, with one exception. Yesterday ( 07 June), for the first time during the second wave, we registered a case count (just under 86,000) lower than the peak of the first wave - this was also the first occasion when the count dipped below six figures. The early hotspots cooled down by mid-May and during the latter half of May, states which peaked slower and later contributed much more significantly to caseload. By now, the epidemic is waning in almost all of India's 36 States and Union Territories. The trajectory in the country as a whole from 10 March until yesterday is shown below. 


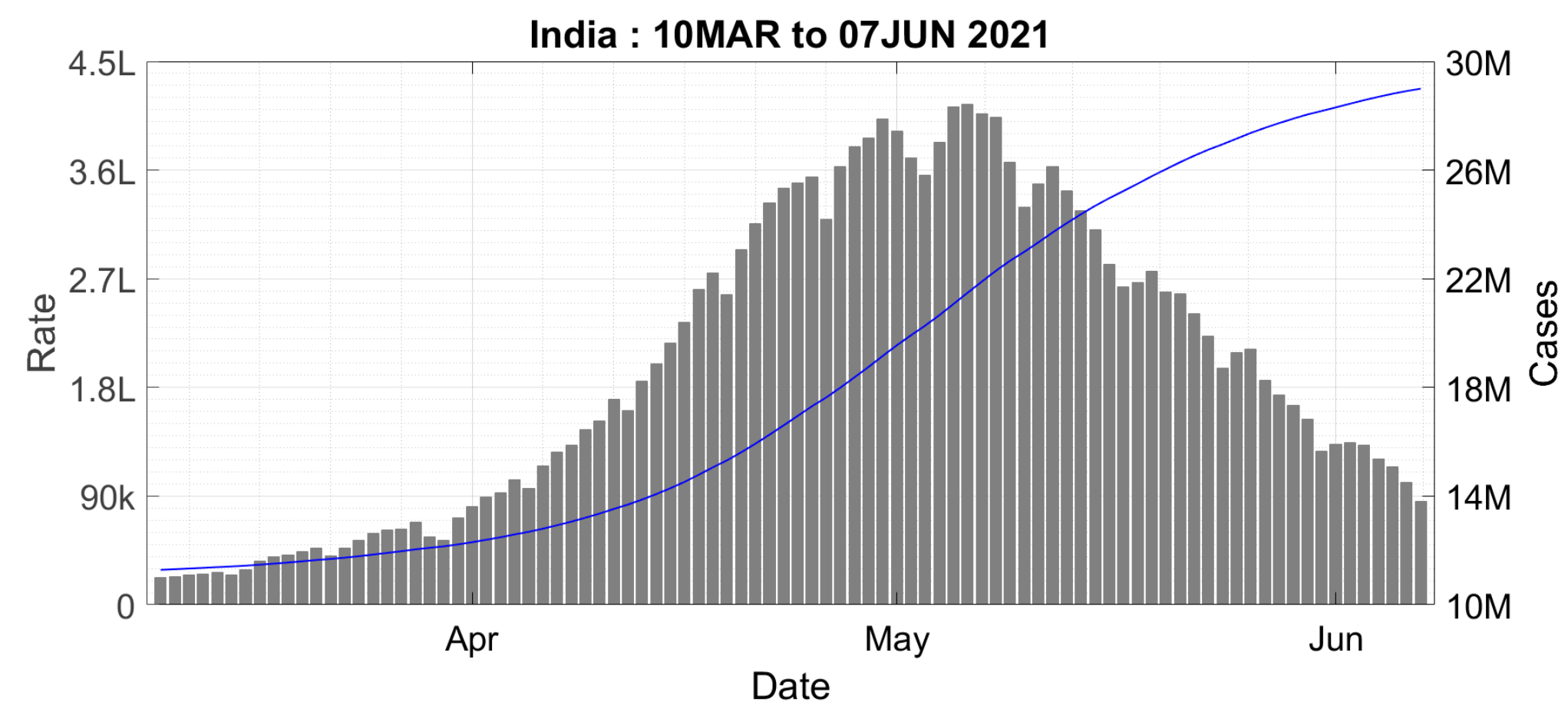

Figure 5 : Case trajectories in India from the approximate start of the second wave until yesterday. The symbol ' $L$ ' denotes lakh and ' $M$ ' million. Labels on the x-axis correspond to the first day of each month.

The burning question is, how did we get here in the first place?

\$2. Current explanations. Conventional epidemiological understanding has us associate a wave of disease with a marked relaxation in precautionary measures. Thus, a sudden lifting of mask mandate might be followed by a case wave, as might a permit returning schools to in-person operation. There was no such transition in the immediate weeks leading up to India's COVID-19 situation. Nor was there an abrupt change in the standards of compliance with NPI. The most anomalous events during the immediately prewave period were the Assembly election rallies and Kumbh Mela mentioned in $\S 1$. Therefore, many reports were quick to ascribe these events as the cause of the second wave in India.

Unfortunately, the epicentre Maharashtra was the site of neither the Kumbh Mela nor any election activity - while it is true that gram panchayat (village governance body) elections had been held in the state, their results had been declared on 19 January 2021 [8], long before the second wave arose. The subsequent hotspots of Delhi and Uttar Pradesh did not have Assembly elections also. In Uttarakhand, following the

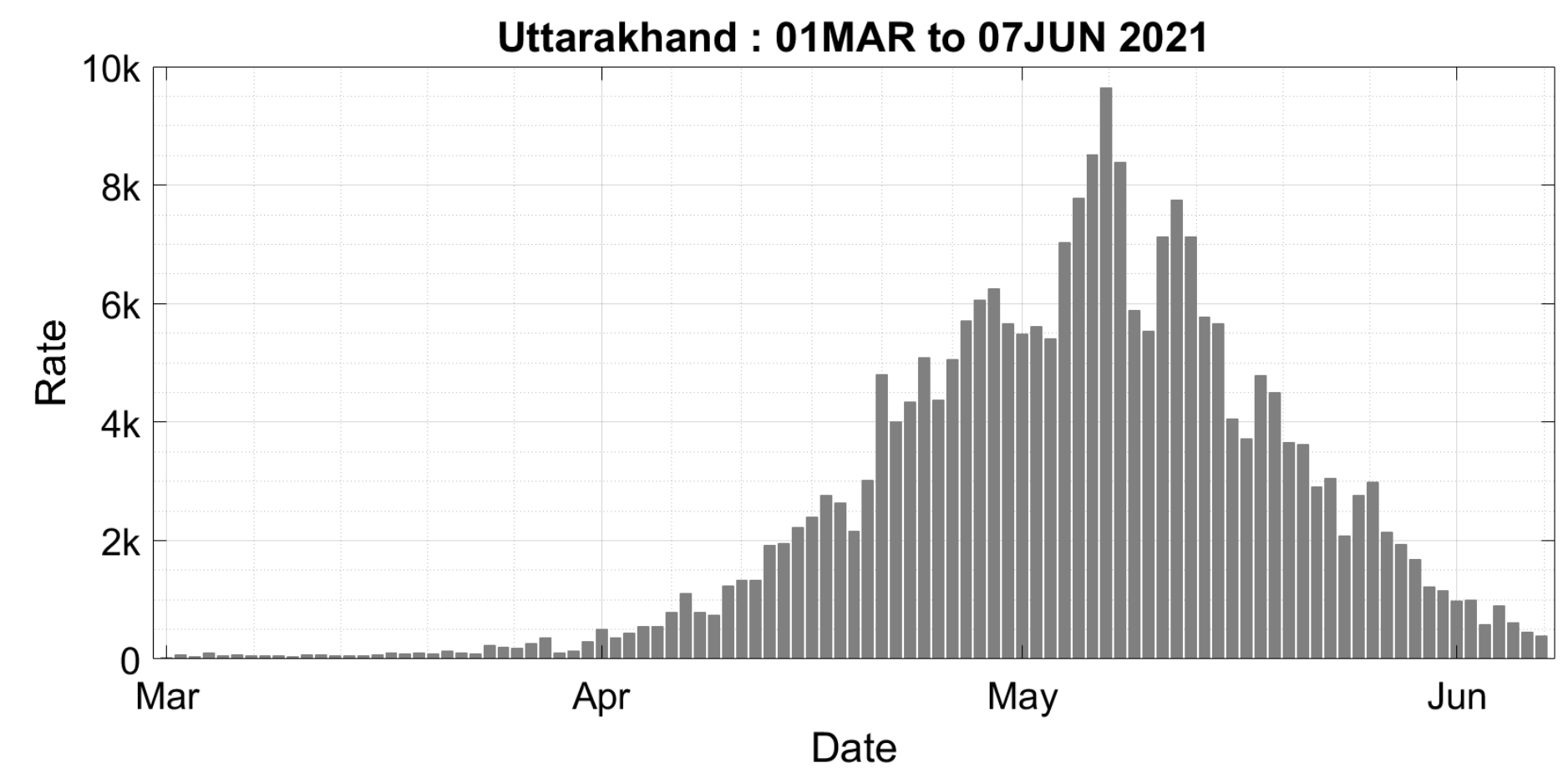

Figure 6 : Case trajectories in Uttarakhand during the second wave. The symbol ' $k$ ' denotes thousand. Labels on the $\mathrm{x}$-axis correspond to the first day of each month. 
truncated schedule, the core of the Kumbh festivities began on 01 April and was slated to continue until the end of that month [9] before being wound down midway. The festival timeline is consistent with the timing of the wave in Uttarakhand, see Fig. 6. The climb and peak (the latter occurring on 07 May) are delayed relative to those of the hotspots shown in Fig. 4, so those case explosions could not have been generated by infected pilgrims returning from Haridwar. Hence, Kumbh and the origins of the second wave are correlated neither spatially nor temporally, and claims of a causal relation between the two are false.

Indeed, the states where political and religious activities have taken place and the states where caseload is high are disjoint sets, to the extent that the worst-hit state in South India was Karnataka while elections had taken place in neighbouring Tamil Nadu and Kerala. The discussion given in articles like Refs. [1013] must be interpreted in context of this fact. The farmers' protest in Punjab has also been mentioned [14] as the root cause of the wave, citing an early increase in cases in that state; while the early start to the Punjab second wave is true, the rise was gradual and the absolute numbers remain low. Punjab was never a hotspot of the epidemic and the evidence to support this hypothesis is also limited. To be clear, we do not for a moment wish to imply that proceeding with Kumbh, election rallies and farmers' protests under the prevailing circumstances was wise. It is just that these decisions did not cause the second wave; the surge would have come and hit us badly even if these erroneous choices had not been made.

Ranjan et. al. [15] and Nesteruk [16] have presented detailed characterizations of the second wave without attempting to explain its origins. A recent study by Juneja and Mittal [17] has focussed on this latter question, using an agent-based model to analyse the second wave in the epicentre Mumbai. After a thorough examination of multiple scenarios related to viral mutation, public behaviour, testing rate etc, these authors conclude that the most probable explanation is the emergence of a hidden mutant (not the double mutant) which had just 2.5 percent prevalence in February but was 2.5 times more transmissible than wildtype. If such a mutant is indeed found, then this hypothesis will be borne out in full. If this mutant is not found and the double mutant continues to remain dominant, then mutation can be a contributing factor to disease burden of the wave but not its root cause.

A very recent study by Dhar et. al. [18] cites the emergence of B1.617 double mutant as the cause of the second wave in Delhi. This is a rigorous formulation of claims made earlier in a more informal context $[19,20]$. Dhar et. al.'s primary basis for their argument is that this strain (and in particular the sub-lineage B1.617.2) became more prevalent relative to all other strains including wildtype and B1.1.7 during the second wave. What this demonstrates incontrovertibly is that the double mutant is more transmissible than all other variants currently circulating in India. As regards the wave's origins, while the increased transmissibility of mutant strain is definitely a factor, we feel that it is not the entire story. Our reasons are as follows.

- Double mutant strain was first discovered in Maharashtra in October 2020. Case counts there and elsewhere decreased for a long time thereafter. Why did the mutant remain dormant for so many months before suddenly initiating uncontrollable spread all over the country? Continuing on these lines, double mutant was introduced to Delhi from Maharashtra as per Dhar et. al. Why are the spreading profiles so different in the two regions?

- As per Fig. 3A of Dhar et. al., wildtype amounted to 30 percent of total cases in March and about 12 percent of total cases in April 2021 in Delhi. Given that there were at least 25 times more cases in April than in March, this implies that wildtype infections also underwent significant growth during the second wave, albeit at a slower rate than double mutant. This is impossible if the mutant is the only factor driving increased transmission.

Pursuant to the above reasoning we conclude that although Dhar et. al. [18] contributes significantly to our understanding of the second wave, it still does not paint a complete picture.

The potential immune escape properties of double mutant are also unlikely to be relevant for the second wave as the overwhelming majority of second wave cases are new cases and not reinfected ones. Convalescent sera [21], Covaxin [21] and Covishield [22] have all been found to possess neutralization activity against this mutant strain. While it is true that neutralization activity is reduced as compared to the 
wildtype, the reduction factor for the vaccines has been found to be mild (2- to 3-fold [21,23] as opposed to 11-fold for B1.351 mutant). Very recently, a study conducted in UK [24] has estimated actual efficacies of Pfizer and Covishield vaccines against the double mutant. This study has found only a small efficacy reduction for both vaccines. Hence there must be some social factors also - not Kumbh or election but something less conspicuous - which resulted in the second wave's assuming the proportions it did.

Summarizing the entire discussion, we can say that the origins of the second wave of corona in India have not yet been explained in a convincing manner.

\$3. Comparison with other countries. While the situation in India is contemporary and, to us as citizens, extremely worrisome, we do not downplay the fact that many other countries have also suffered huge waves of outbreak with sudden notice and that in each case, the consequences of the biggest wave have been tragic. In Table 1 we display the successive maxima and minima in the case trajectories of India as well as several other countries. The data for countries other than India are taken from Microsoft's COVID-19 tracker [25]. From these data sets, we can see that multiple waves characterize the epidemic dynamics in many countries, on a wide spectrum of economic advancement.

\begin{tabular}{|c|c|c|c|c|c|c|}
\hline Trajectory & & India & Argentina & Germany & Pakistan & USA \\
\hline \multirow{2}{*}{$\operatorname{Max} 1$} & Date & 16SEP2020 & $18 \mathrm{OCT} 2020$ & 02APR2020 & 14JUN2020 & 08APR2020 \\
\hline & Case rate & 97,860 & 15,051 & 5596 & 5866 & 31,415 \\
\hline \multirow{2}{*}{ Min 1} & Date & 01FEB2021 & 07DEC2020 & 11JUN2020 & 30AUG2020 & 07JUN2020 \\
\hline & Case rate & 8579 & 4956 & 327 & 340 & 19,181 \\
\hline \multirow{2}{*}{$\operatorname{Max} 2$} & Date & 06MAY2021 & 07JAN2021 & 12DEC2020 & 05DEC2020 & 19JUL2020 \\
\hline & Case rate & $4,14,280$ & 11,712 & 25,851 & 3300 & 66,184 \\
\hline \multirow{2}{*}{ Min 2} & Date & FUTURE & 13FEB2021 & 16FEB2021 & 09FEB2021 & 08SEP2020 \\
\hline & Case rate & । & 5436 & 7090 & 1214 & 33,393 \\
\hline \multirow{2}{*}{$\operatorname{Max} 3$} & Date & | & 20APR2021 & 23APR2021 & 20APR2021 & 07JAN2021 \\
\hline & Case rate & | & 24,017 & 21,000 & 5694 & $2,57,732$ \\
\hline \multirow{2}{*}{ Min 3} & Date & । & FUTURE & FUTURE & FUTURE & 15MAR2021 \\
\hline & Case rate & | & | & | & | & 47,901 \\
\hline
\end{tabular}

Table 1 : Maxima and minima of daily case rates in five countries. For the countries other than India we have used the seven-day averaged figures from Ref. [25]. In Argentina, Germany and Pakistan, cases are currently decreasing after reaching Max 3 while USA climbed from Min 3 to about 75,000 cases in early April before again posting a decline, this last very likely brought on by vaccination.

If the country's population is higher, the absolute numbers tend to be larger. Thus, India's record high numbers are partly the result of its huge population rather than an astonishingly high disease prevalence. Some parameters for India are however unusually large by any standards. For example, the height of the wave relative to the minima is huge - the ratio of Max 3 to Min 2 is about 8 in USA while Max 2 to Min 1 is about 50 in India (the ratio is very high in Germany as well). Given a serial interval of five days [26,27], the reproduction number $R$ (defined as the number of people to whom one case transmits the disease on average) during the second wave in Delhi works out to 1.8 and in Lucknow to 2.1 or more; such values are unheard of for anything but the initial wave of COVID-19 anywhere in the world. The high values of both these metrics have been responsible for the acute shortages of medical facilities - healthcare systems were 
completely blindsided by the suddenness and severity of the wave. There was no prior evidence, either theoretical or experimental, which would have prepared hospitals for a 30- to 100-fold increase in bed, ventilator or medical oxygen requirements over a month (just look at Fig. 4-mid and 4-bot again - they represent a steep, or even insurmountable, challenge for any healthcare system anywhere in the world).

As examples not in Table 1, Uruguay, previously hailed as a model in COVID-19 control, started undergoing its first wave from February 2021. This wave, which is still continuing at undiminished ferocity, is of considerable size and has overstressed healthcare systems. Beginning in late March, Japan exhibited a fourth wave which, for the first time in its pandemic history, resulted in patients not finding hospital beds and passing away at home [28]. This wave has put the Olympic Games under threat. (Again, during this wave, the worst hit city of Osaka had a peak of about 1200 daily cases, increasing from a tenfold lower baseline in about three weeks. If this can cause a healthcare crisis in Japan, then we can imagine the havoc that Fig. 4 will wreak in Delhi and Uttar Pradesh.) Taiwan had nearly zero daily case counts all the way from January 2020 upto May 2021, but it is now accumulating more cases in two days than it did in the past year. Fatalities, almost absent upto last month, are also accruing at a worrisome rate.

In many countries, the daily case rate shows an extended region of roughly constant behaviour near the minima. This is especially true for India (Min 1, see Fig. 3 as well as Fig. 4 for individual regions), Germany (Min 1) and USA (both Min 1 and 2). Taiwan, prior to its wave, may be considered as an extreme example. This constant rate behaviour appears to continue for an extended period, breeding a false sense of security before a wave rushes in and catches healthcare systems unprepared. These similarities in spreading profile across the globe suggest that the Indian wave was caused not by something unique to the country but by a more universal yet hitherto unknown infectious disease dynamical phenomenon.

\$4. Existing mathematical model predictions. COVID-19 transmission models can broadly be divided into four classes : (a) lumped parameter or compartmental models, (b) agent-based models, (c) stochastic differential equation models and ( $d$ ) data-driven models. Among these, the first two approaches are the most widely used. Traditional lumped parameter models are based on ordinary differential equations (ODE) such as S-I-R and S-E-I-R (of which Ref. [5] as well as influential studies conducted in other countries [29-33] are extensions). Such models have only a finite number of fixed points where the epidemic can end; because of this, it is very difficult to pass a peak unless a significant population fraction has already been infected. A novel compartmental model based on delay differential equations (DDE) has recently been proposed by our group [34] - in this model, every infection level is a possible fixed point so the above drawback is avoided. In all these models however, a constant case rate is not a generic solution; it occurs only if $R$ equals exactly unity, which happens for specially chosen parameter combinations and infection levels. These models do have second waves corresponding to reopening but their origins are not baffling [15], like the India wave. For example, in the DDE model, $R$ at any time is given by the product of the average daily interaction rate, the transmission probability, the susceptible population fraction and the transmissibility duration. A second wave occurs if during a reopening process $R$ changes from below unity to above unity. To bring about a sharp wave as in the Indian case, the change in $R$ must be significant as well as rapid. This implies a quick and strong increase in interaction rate or spreading probability, which can only be brought about by an act of policy (release of lockdown, revocation of mask mandate etc). The absence of such a phenomenon in India in February-March has led to fallacious theories being proposed, as we have discussed previously.

Among agent-based models, some efforts from the UK $[35,36]$ are commendable but appear to have missed the origin of the waves, at least in a general setting. Most remarkable among the existing Literature is the study by Thurner et. al. [37]. This is the first work (and one of a handful so far) to have found a constant case rate as a stable, generic solution of a disease transmission model. We have discussed this model in detail in Ref. [1]; the bottom line is that it casts no new insight into the origins of COVID-19 waves beyond the existing compartmental models. The same holds true for a few other studies reporting the constant rate solution [38-40].

The works we mentioned in this brief review are the most unique on account of either the influence they have exerted or the novelty of their content. These apart, there have been several thousand corona modeling 
studies posted and published during the past year. Among all these studies - ours included - there was, to the best of our knowledge, zero evidence to suggest that the pandemic situation in India in March to May might become what it is given that social mobility and case counts in October to February were what they were.

\$5. Purpose of the study. The International Civil Aviation Organization (ICAO) [41] provides a key to the constructive analysis of any catastrophic event : "The sole objective of the investigation of an [aircraft] accident or incident shall be the prevention of accidents and incidents. It is not the purpose of this activity to apportion blame or liability." We are independent investigators and our singular purpose in embarking on this study is to understand what went wrong so that a repetition may be averted both in India and abroad.

\section{MODEL AND PREDICTIONS}

\$6. Summary of mathematical model and results. In a companion work [1] we have proposed a mathematical model which overcomes the deficiencies of the studies mentioned in $\S 4$ and is capable of explaining the second wave in India. Since the model is perfectly general i.e. applicable to all countries (and since length was becoming an issue), we have decoupled it from the present study which we treat as the first detailed application of the model. In this Section we present a summary of the model and the results which it yields. The entire modeling study is available at the following link:

\section{https://www.medrxiv.org/content/10.1101/2021.06.02.21258243v1}

and we urge you to consult the same for the details.

In Ref. [1] we have ignored vaccination since at the time of onset of the second wave, only a small fraction of India's population had been vaccinated. (This is another unfortunate consequence of our unwieldy population - a moderate infection prevalence causes a huge case count while a fast vaccination drive achieves only limited coverage.) It is definitely true that the preferential vaccination of healthcare and frontline workers as well as senior citizens, i.e. the populations at highest risk of exposure and/or mortality, has averted hundreds or even thousands of deaths per day. However, in both Ref. [1] and here, we focus on cases only, and we leave the calculation of mortality averted by vaccination drive for future analysis.

The model in Ref. [1] starts from two key hypotheses :

- The majority of transmission occurs during close, unmasked interactions between a case and a potential target.

- When entertainment venues such as restaurants and cinema halls are open but COVID-appropriate behaviour remains in place, then such interactions are primarily confined within small, closed groups of family members and friends which we call clusters.

Cluster transmission, wherein the virus remains trapped within small sub-populations instead of diffusing freely through the entire population, can qualitatively explain how it is possible for case counts to remain low even with a relatively high level of socioeconomic activity. In Ref. [1] we have identified two mechanisms of transmission across clusters. The first is unintentional cluster transition (UCT), which takes place during activities like shopping, travelling to work etc. UCT occurs when there is a protocol violation (such as mask or separation fault) or an instance of bad luck (for example, the virus jumping across masks) in such an environment. It also includes transmission from objects, which is believed to be much rarer than inter-person transmission. The second mechanism for inter-cluster transmission is socializing external to cluster (SEC) which encompasses events such as marriage functions and celebratory parties. Venues of this kind feature guests who normally do not socialize with each other, for example multiple branches of extended families as well as mutually-stranger bride and groom's friends all invited to a wedding. At SEC events, adherence to COVID-appropriate behaviour tends to be low and there is considerable pressure to actively interact with people outside one's immediate circle. 
The model we have constructed is discrete-time, discrete population and deterministic. It takes the form of an algorithm which is run on a computer. It assumes homogeneous mixing at the cluster rather than the individual level and hence is applicable to a city and not a larger region such as a state or country. The key parameters are summarized in Table 1 below.

\begin{tabular}{l|r|}
\hline Parameter & $\begin{array}{r}\text { Significance } \\
\text { Total population }\end{array}$ \\
$n_{U}$ & Number of people participating daily in UCT events \\
$P_{U}$ & Probability that case transmits disease to target at a UCT event \\
$n_{S}$ & Number of people participating daily in SEC events \\
$m_{S}$ & Number of targets infected by one case at a SEC event \\
\hline
\end{tabular}
Table 1: The most important parameters in the mathematical model. For a full list of parameters and their values,
leasult Ref. [1].

We have found that for constant $n_{U}$ and $m_{S}$ and zero or small $n_{S}$, there is a range of $P_{U}$ for which the epidemic continues for a long time at approximately constant daily case rate before eventually dying out. We call this the default solution. We have further identified three effects which are of importance in the management of the epidemic. These are

- Classical instability. Starting from the default solution, if $P_{U}$ is increased above an upper cutoff while keeping other parameters unchanged, then the default becomes unstable and gets replaced by a wave. (When $P_{U}$ is decreased below a lower cutoff, the default gets replaced by one where the epidemic is eliminated in time but, for the disease in question, that solution belongs to the fantasy world.) This instability is also present in classical epidemiological models, hence the name. With respect to epidemic management, this is the most tractable of the instabilities present in the model since the response of the system to a small change in parameters remains small.

- Cryptogenic instability. All other parameters remaining constant, an increase in $n_{S}$ above a cutoff value destabilizes the default solution into a wave. We call this instability cryptogenic because the increase in $n_{S}$ causes a negligible change in the population-averaged contact rate, which cannot lead to a stability transition in classical models. Once the instability occurs, the speed and severity of the wave increase very rapidly with increasing $n_{s}$. It does not matter whether a single gathering of size $n_{S}$ people or fifty separate gatherings amounting to total $n_{S}$ people is/are taking place. The high sensitivity of the epidemic curves to change in parameters makes this phenomenon dangerous from the epidemic management perspective.

- Critical mass effect. Even when the parameter values are chosen such as to generate an instability, a very small case rate might prevent it from actually being manifest. For example, in one set of simulations in Ref. [1] (Fig. 4 of that Reference), we have considered an unstable parameter combination in a model city of population just above one lakh. What we have varied is the initial number of cases which are introduced externally to start off the outbreak. With 7 initial cases spread over 3 days, we have found zero further infections in the local population. Replacing 7 by 17 has led to 138 additional infections over the next 80 days. Replacing 17 by any number greater than 20 has led to 73,000 infections over the next 500 days. This phenomenon is extremely dangerous from the viewpoint of epidemic management as it implies that if the case counts are very low then the instability will not be visible and the authorities will not even be aware that it exists. The converse of this phenomenon does not hold true however - if the parameters are chosen to generate a constant/decaying solution, then even a huge initial condition cannot make the epidemic perpetuate on its own. This last fact agrees with conventional wisdom. 
With this, we conclude our summary of the model and results of Ref. [1]. We again recommend to please consult that Reference for questions about any details we have not explained here. Now, taking the model and the results for granted, we show how they explain the origins of the second wave in India.

---- 0 ----

\section{DISCUSSION}

\$7. Explanation of the second wave in India. Before starting this discussion, we highlight that the parameter values used for the analysis in Ref. [1] are indicative only. Rather, given the basic structure of the model, the phenomena of classical and cryptogenic instability and critical mass effect are expected to be robust across parameters, and these phenomena help us understand the dynamics of COVID-19 spreading in India. So far, we have not attempted a fit of data from India or any state to this model, if for no other reason than that any data set can be fitted to any model whatsoever. Given this disclaimer, we present our conjecture of what happened in India between March 2020 and May 2021. We use the jargon "super-stable", "stable" and "unstable" to indicate operating points in the model parameter space corresponding to elimination, default solution and wave respectively.

During the initial lockdown (March to June 2020), the spread of the virus was not stopped but proceeded very slowly. This implies that elimination of the pandemic (Fig. 2-top in Ref. [1]) is impossible to achieve in the Indian context. This is not a surprise - hard lockdowns failed to halt transmission anywhere except in the most sparsely populated countries of Oceania and certain countries of East Asia where people might have innate immunity against the pathogen. During the lockdown, India was probably marginally unstable, which increased to definitely unstable as the unlocking procedure began, for economic reasons. Nevertheless, due to tight implementation of NPI, the instability was mild (as in Fig. 2-bot of Ref. [1]). The case rate increased very slowly until the accumulated infections made the operating point become stable in mid-September. From this point onwards, the rates decreased gradually while society remained largely open. By January 2021, India was operating in the stable mode like the default solution with a low and approximately constant daily case rate.

We now explain the origin of the second wave in Maharashtra with no apparent cause. In February-March 2021, the paradoxically low level of virus given the high level of reopening, together with the added mental security arising from the vaccines, provided a stimulus for increase in the number of wedding ceremonies, parties and similar social gatherings which had remained suspended for so long. SEC transmission and the cryptogenic instability mechanism being unknown at this time, neither the authorities nor the public were opposed to such functions' taking place. We conjecture that at some time in February, Maharashtra crossed the threshold of the cryptogenic instability. It is logical that when a stability threshold is breached, the cases will increase only slowly at first, with the increase picking up as the region becomes more and more unstable. This is consistent with the behaviour observed in Mumbai and Pune where a slow rise in case rate can be seen even from the middle of February (see Fig. 4-top for the graph of the state as a whole). At that time of course, the increase was dismissed as just another local fluctuation, which common sense teaches us to expect in any dynamics of this nature.

While cryptogenic instability can explain the dynamics of Maharashtra, it alone cannot account for either the almost-but-not-quite synchronous rise of cases in Delhi and Uttar Pradesh, or their astronomical growth rate starting from a nearly zero baseline (see Fig. 4 and caption for the differences between Maharashtra and the other two regions). It is inconceivable that different regions will all make their cryptogenic stability transitions within a week of each other, and that the subsequent hotspots of Delhi and Uttar Pradesh will proceed overnight from stable to explosively unstable (unless by official mandate, which was not the case). To resolve this discrepancy, we invoke the critical mass effect. We believe that, during February-March, Delhi and the main cities of Uttar Pradesh like Lucknow, Kanpur and Allahabad had already made their transitions as a result of increase in social functions. However, the absolute case counts there were so low that the instability did not manifest itself after it occurred and we kept seeing low infection prevalence. 
Perceiving no threat, caution continued to be relaxed (increase of $n_{S}$ and probably of $P_{U}$ as well) and the operating point in these states drifted farther and farther into the danger zone.

For Delhi, the critical mass very likely came from Mumbai [18]. Delhi and Mumbai are the pair of cities in India with by far the maximum number of connecting flights (the route is in fact the fifth busiest in the world [42]). When cases went up in Mumbai, passengers almost certainly arrived in Delhi with the virus and spread it into the local community. Now, the illusory stability was shattered and the true nature of Delhi's operation was revealed as extremely unstable, so much so that $R$ had the value $1 \cdot 8$. The rise in Uttar Pradesh can be explained in a similar manner. This state is contiguous to Delhi and has copious rail and road links with it - operating way beyond the stability threshold, it merely needed an influx from Delhi to achieve critical mass and proceed almost immediately to detonation.

The increase in cases all over India during the second wave can be explained by a combination of these two phenomena as well. As we have mentioned in $\$ 6$, external cases introduced into stable regions cannot cause a runaway outbreak - in March-April 2021 the bulk of India appeared to be stable and should have resisted the external influx from a few isolated hotspots. In all probability however, by February, almost the entire country had sunk to a case rate below the critical mass to activate a wave in case of instability. During this time, many cities had made their stability transitions, either classically or cryptogenically or both, but were unaware of the fact. However, when the first few epicentres got activated, travel links ensured that quantities above the critical mass were seeded almost everywhere. Thereafter, the growth rate in each state was determined by how unstable it was to begin with - states like Gujarat and Tamil Nadu witnessed a slower increase (about 10 times in one month) because they were operating in a less unstable mode while states like Chhattisgarh and West Bengal witnessed a faster increase (about 25 times in one month) because they were operating in a more unstable mode.

Why didn't the mini-waves of Fig. 2 lead to a national disaster like the second wave? We conjecture that there are three reasons. Firstly, the festival season was in October-November, when case rates were high. This ensured that seeding was always above critical mass and an increase began as soon as the stability threshold was breached. We have already argued that such an increase must initially be gradual; the authorities, already on alert, reacted immediately to quell the surge. Secondly, due to prevailing awareness of the situation, the festivals probably did not lead to too many SEC gatherings. Rather, people celebrated with their friends and family and paid more visits to entertainment venues and shopping malls. Other than facilitating intra-cluster transmission, these activities would cause an increase in UCT events which, as mentioned in $\S 6$, leads to only a mild instability and is easier to control. Thirdly, regions apart from the localized hotspots were operating in the stable mode so the influx of cases from the hotspots (which must have occurred since travel links were open) did not initiate spiralling outbreaks anywhere else.

Extreme complacency regarding the management of the disease arose during January-February 2021 likely because of the astonishingly low disease prevalence despite the high social mobility. The successful management of the festival-related mini-waves also gave rise to a deceptive sense of confidence. No prior mathematical modeling study had fully characterized the default solution, in particular the circumstances when it can occur and the instabilities which it can suffer. In all classical formulations (including Ref. [34]), low case rate despite high interaction rate can only be achieved when a sufficient fraction (depending on transmissibility and susceptibility) of the population has been infected. Such reasoning motivated the conclusion of herd immunity [5] and in turn led to a widespread perception that the threat of COVID-19 was past.

As modellers, what was our own personal opinion on the situation during January-March 2021 ? It was that somehow Indians must be intrinsically less susceptible to the coronavirus than people of some other races. As a result, $R$ must have been lower for India to begin with, and the few cases which had occurred during the first wave (together with the unreported infections) were sufficient to bring it down below unity. As case counts became really low, they passed below the threshold of validity of the continuous model [34] and we started seeing the constant solution reported by Thurner et. al. [37], which we believed would continue until the disease's eventual elimination. Despite a yearlong association with COVID-19 modeling, we too had been completely blind to the threat of a second wave in April-May. 
The notion of India without corona triggered a sharp increase in SEC events like weddings, parties etc and, as we have already seen in $\S 1$, also motivated the decisions to hold full-scale Assembly elections and Kumbh Mela and continue with the farmers' protests in Punjab. While the trigger for the second wave came from the social functions (the organized gatherings having been shown in $\S 2$ to be uncorrelated), these large gatherings definitely added to the burden of disease in their respective states once the wave arose. For example, without the election activity, West Bengal might have witnessed a 10-fold rather than a 25 -fold case rise in a month after being seeded from outside with the critical mass. Similarly, if Kumbh had not been held, Uttarakhand might have experienced a 10- or 20-fold increase of cases from the baseline rather than the almost 100-fold increase which it actually underwent (see Fig. 6). The pilgrims, though by no means the cause of the second wave, are certainly to be numbered among its victims.

A similar situation holds true for the B1.617 double mutant and the B1.1.7 mutant. A highly transmissible variant will lead to increases in $P_{U}$ as well as $m_{S}$ and will facilitate both classical and cryptogenic instabilities. Since the double mutant did not initiate uncontrolled growth between October and January, we conclude that it is not transmissible enough to generate instability at the reopening level prevalent in those months. As discussed in $\$ 2$, genomic sequencing data suggests that it alone cannot be called the proximal cause of the wave. It is true however that with a higher transmissible mutant, the stability boundary will be breached at a lower threshold value of $n_{S}$ and conversely, a given value of $n_{S}$ will generate a stronger instability. Thus, B1.617 double mutant definitely had a role in facilitating the instability transitions and exacerbating the disease burden once the critical mass was seeded into unstable regions.

Is it possible to estimate the relative transmissibilities of two or more concurrently circulating mutant strains during a phase of constant case rate? The answer may be yes, and maybe no. This is because we get the default solution for a whole range of $P_{U}$, even though this parameter is directly related to transmissibility. If a less-transmissible mutant happens to have its effective $P_{U}$ below the cutoff for the constant solution, then that will get dominated by the other mutant/s. If however all the mutants have their effective $P_{U}$ within the default range, then they will all evolve at approximately constant rates, give or take the stochastic fluctuations. In this case, domination by any one strain will not take place. Once a wave is initiated however, all the mutants which undergo exponential growth will find their prevalences sorted in order of transmissibility - the more infectious the strain, the higher will be its growth rate. Any mutant which is so less transmissible as to not enter exponential growth will automatically be reduced to nearly zero prevalence. This is in line with the findings of Ref. [18] which clearly mentions that during February and even March 2021, the prevalence of B1.617 and its sub-lineages in North India was quite small, and it became the dominant strain only in April. It also disproves beyond doubt any claims about the second wave's having been predicted in advance and caused as a result of wilful negligence.

It is very likely that the waves in other countries (\$3) were also the result of similar phenomena. The question arises as to why the second waves in many of these countries were less severe as compared to India. Paradoxically, this might be an upshot of the better management of corona in India during the first wave than in many other countries. As Table 1 shows, the minima following the first (or second) waves in many countries are less pronounced than in India - this might have ensured that those countries always remained above critical mass and hence responded quickly to instability, as happened for the Indian miniwaves of Fig. 2. India's better management of the first wave was probably due to initially greater adherence to NPI than in other countries. By achieving compliance over an extended period, India managed to drive the first wave down to below the critical mass, a fact which tragically became its undoing. Such a phenomenon might have happened in Germany also, where after months of very low case counts following the excellent management of the first wave, the second wave arose with considerable ferocity. Uruguay and Taiwan, where corona was nowhere until it was everywhere, are very likely similar cases. We hypothesize that the critical mass effect occurred in these countries as well, although we currently need more data regarding social mobility there to substantiate this claim.

§8. Recommendations. Localized NPI such as full lockdown, weekend lockdown and/or evening curfew are in place in almost every State and Union Territory. These measures have certainly been effective as the nationwide case rate has reduced by approximately four times from the peak value. The rates in the 
early hotspots have reduced by higher factors and the dominant contribution to the total caseload now comes from other states. This development is extremely positive as it implies that the double mutant is not so transmissible as to respond only weakly to a strong NPI. Hard lockdown is impractical to continue for extended duration but with a renewed focus on mitigation measures as was the case in October 2020, India should be able to re-enter a stable and open mode of operation within the end of this month.

Once control over the virus is re-established but vaccine-induced herd immunity is far away, it is vital that a third national wave be avoided. Now that the spreading dynamics of the disease are better understood, this should prove feasible. To mitigate SEC transmission, size limits on weddings and parties have to be chosen conservatively and enforced strictly. Public health messaging should focus on explaining why these restrictions are necessary so as to elicit people's willing cooperation. Citizens should also be urged to consciously confine socializing and entertainment activities within a fixed set of close contacts - this should be more appealing than having to discard recreation altogether. Organized gatherings like Kumbh Mela should of course be scrapped or curtailed in size; where cancellation of events is impossible due to popular sentiment, mask and separation mandates must be enforced rigorously. Just as importantly, masking should be enforced in long distance trains; the extended journeys and the compartments of size 4 to 8 within each coach are ideal venues for interaction among different clusters and hence for SEC transmission. Coach capacity reductions - for example allowing only one family to travel in one compartment, cabin or coupe - might also help to mitigate in-train transmission. Genomic sequencing should be prioritized so as to monitor for the emergence of mutant strains. Activities which might be linked to mutagenesis, such as convalescent plasma therapy or other therapy in immunocompromised or unresponsive patients [43-45], must be undertaken with extreme caution. Finally, local outbreaks in any region should be treated as potential emergencies instead of being brushed off as inevitable fluctuations; to avoid critical seeding in any city which might happen to be unwittingly unstable, travel from such regions must be suspended or monitored until the outbreak is brought under control.

The only long-term exit option from the pandemic is vaccination. After a pronounced slump in the month of May, the vaccination drive in India is again showing definite signs of accelerating. We are aware that scale-up of vaccine production while ensuring quality and safety is complex and time-consuming and are confident that, after the recent experience, the Central and State Governments as well as the vaccine manufacturers are working to ensure the fastest possible rollout. The conclusion of the study [24] that two doses of Covishield offer significantly better protection against the double mutant than one dose is noteworthy in view of the decision to extend the Covishield dosing interval to 12-16 weeks. It should be attempted to repeat the study in India and use the results to decide on a suitable interval.

In a recent study [46], we have used a classical model to predict the effects of selective relaxation of restrictions for vaccinated individuals. We have found that such a policy is permissible if the vaccines are above a certain cutoff efficacy. In the near future we shall repeat the study using the more accurate model of Ref. [1]. Right now however, relaxation of restrictions for anyone is still some time away. Vaccines and NPI have to work in tandem to reduce the disease prevalence as quickly as possible.

\$9. Conclusion and future directions. To summarize, we believe that the primary driver of the second wave of COVID-19 in India was the cryptogenic instability arising from social gatherings like weddings and parties and the critical mass effect arising from the effective management of the first wave. The combination of these effects enabled multiple cities to cross the stability threshold with no warning and they were all hit soon after the first epicentre (Maharashtra) was activated. The emergence of double mutant strain and activities such as Kumbh Mela and electioneering in multiple states did not result in the origination of the wave, but they did increase the disease burden once the wave already arose. Thus, we posit that the wave was caused by our collective lack of knowledge of vitally important phenomena pertinent to infectious disease dynamics while its effects were exacerbated by mutant strain and complacency regarding the future destructive capacity of the disease.

April 2021 contained the worst moments for India in its struggle against the coronavirus pandemic. Coming like a bolt from the blue, the tsunami of cases has left many people rattled and in fear of a third wave. Part of the uncertainty stems from the lack of a comprehensive understanding of the factors which 
triggered this catastrophe. We hope that our analysis can provide reassurance as well as guidance. With status quo prevailing on the mutation front, localized mini-waves like in Fig. 2 are probably unavoidable but a synchronized national wave might not occur again so long as caution prevails until most people are vaccinated.

The analogy with aircraft safety investigations mentioned in $\$ 5$ is again relevant. In the early years of commercial aviation, many dynamical phenomena related to flying were unknown and came to light only in the worst possible manner i.e. through airplane crashes. Every tragedy provided a learning experience [47] and, with time, commercial flying developed into the nearly risk-free enterprise which it is today. The same holds true for infectious disease modeling and management. COVID-19 is the first pandemic where humanity is equipped with the scientific and technological tools to attempt predicting and preventing its spread. Gaps in our knowledge are inevitable, and their consequences are terrible. The best we can do is to learn from our mistakes and prevent reruns of this phenomenon both in India and the world.

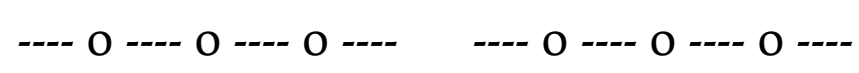

\section{DECLARATIONS}

We have NO conflict of interest to declare.

We have NOT been funded by any agency, public or private, to conduct this investigation.

\section{REFERENCES}

[1] B Shayak and MM Sharma, "A Cluster-based model of COVID-19 transmission dynamics," MedRxiv Article (2021). Available at https://www.medrxiv.org/content/10.1101/2021.06.02.21258243v1

[2] https://www.covid19india.org/

[3] https://www.gisaid.org/hcov19-variants/

[4] https://www.iith.ac.in/ m vidyasagar/research.html\#COVID

[5] M Agrawal, M Kanitkar and M Vidyasagar, "SUTRA: An Approach to Modelling Pandemics with Asymptomatic Patients, and Applications to COVID-19," Arxiv Article (2021). Available at https://arxiv.org/abs/2101.09158. Version 2 is the version which was uploaded prior to the second wave and to which we have referred. Version 3 was uploaded by the authors after the second wave, which has been attributed to mutation of the virus and people becoming careless.

[6] https://www.euttaranchal.com/tourism/kumbh-mela-haridwar.php

[7] https://swarajyamag.com/insta/we-are-in-the-end-game-of-covid-19-pandemic-dr-harsh-vardhan

[8] https://www.timesnownews.com/india/article/maharashtra-gram-panchayat-election-result-2021-latestupdates/708499

[9] https://www.indiatoday.in/coronavirus-outbreak/story/haridwar-kumbh-mela-people-openly-flout-covid19-protocolsground-report-1790552-2021-04-13

[10] The Editor/s of The Lancet, "India's COVID-19 emergency," The Lancet 397 (10286), 1683 (2021)

[11] S Manik, S Pal, M Mandal and M Hazra, "Effect of 2021 Assembly Election in India on COVID-19 transmission," MedRxiv Article (2021). Available at https://www.medrxiv.org/content/10.1101/2021.05.30.21258040v1

[12] A Visaria, P Polamarasetty, S Reddy et. al., "Characterization of the second wave of the COVID-19 pandemic in India : a Google Trends analysis," MedRxiv Article (2021). Available at

https://www.medrxiv.org/content/10.1101/2021.05.19.21257473v1

[13] S Kumar, "Second wave of COVID-19 : emergency situation in India," The Journal of Travel Medicine (2021)

[14] VG Narayanan, "Data story : did the farmers' protest cause the second wave in India ?" (2021). Available at https://swarajyamag.com/politics/data-story-did-the-farmers-protest-cause-the-second-wave-of-covid-19-in-india [15] R Ranjan, A Sharma, and MK Verma, "Characterization of the Second Wave of COVID-19 in India," MedRxiv Article (2021). Available at https://www.medrxiv.org/content/10.1101/2021.04.17.21255665v2

[16] I Nesteruk, "The COVID-19 pandemic storm in India," MedRxiv Article (2021). Available at https://www.medrxiv.org/content/10.1101/2021.05.06.21256523v1

[17] S Juneja and D Mittal, "Modelling the Second Covid-19 Wave in Mumbai," Arxiv Article (2021). Available at http://arxiv.org/abs/2105.02144

[18] MS Dhar, R Marwal, VS Radhakrishnan et. al., "Genomic characterization and epidemiology of an emerging SARS-CoV-2 variant in Delhi, India," MedRxiv Article (2021). Available at 
https://www.medrxiv.org/content/10.1101/2021.06.02.21258076v1

[19] https://www.newindianexpress.com/nation/2021/may/06/double-mutant-tied-to-surge-3rd-wave-inevitable-centre2298939.html

[20] R Guleria, "Why did India have a second wave," (2021). Available at

https://economictimes.indiatimes.com/industry/healthcare/biotech/healthcare/covid-second-wave-aiims-director-drrandeep-guleria-lists-two-main-reasons-for-huge-surge-in-cases/videoshow/82118603.cms?from=mdr

[21] PD Yadav, GN Sapkal, P Abraham et. al., "Neutralization of variant under investigation B.1.617 with sera of

BBV152 vaccinees," BioRxiv Article (2021). Available at

https://www.biorxiv.org/content/10.1101/2021.04.23.441101v1

[22] PD Yadav, GN Sapkal, P Abraham, G Deshpande and DA Nyayanit, "Neutralization potential of Covishield vaccinated individuals against B.1.617.1," MedRxiv Article (2021). Available at

https://www.biorxiv.org/content/10.1101/2021.05.12.443645v2

[23] M Hoffmann, H Hofmann-Winkler, N Krueger, and A Kempf, "SARS-CoV-2 variant B.1.617 is resistant to Bamlanivimab and evades antibodies induced by infection and vaccination," BioRxiv Article (2021). Available at https://www.biorxiv.org/content/10.1101/2021.05.04.442663v1

[24] JL Bernal, N Andrews, C Gower et. al. "Effectiveness of COVID-19 vaccines against the B1.617.2 variant," MedRxiv Article (2021). Available at https://www.medrxiv.org/content/10.1101/2021.05.22.21257658v1

[25] https://www.bing.com/covid/

[26] H Nishiura, NM Linton and AR Akhmetzhanov, "Serial interval of novel coronavirus (COVID-19) infections," International Journal of Infectious Diseases 93, 284-286 (2020)

[27] B Rai, A Shukla and LK Dwivedi, "Estimates of serial interval for COVID-19 : a systematic review and metaanalysis," Clinincal Epidemiology and Global Health 9 (1), 157-161 (2021)

[28] B Guha Majumder, "Japanese are dying at home," (2021). Available at https://swarajyamag.com/newsbrief/japanese-are-dying-at-home-slow-vaccination-and-more-concerning-covid-19-variants-carry-the-blame [29] R Li, S Pei, B Chen et. al., "Substantial undocumented infection facilitates the rapid dissemination of novel coronavirus (SARS-CoV-2)," Science 368 (6490), 489-493 (2020)

[30] G Giordano, F Blanchini, R Bruno et. al., Modelling the COVID-19 epidemic and implementation of populationwide interventions in Italy, Nature Medicine 26 (6), 855-860 (2020)

[31] RM Anderson, JAP Heesterbeek, D Klinkenberg and TD Hollingsworth, "How will country-based mitigation measures influence the course of the COVID-19 epidemic?," The Lancet 395 (10228) 931-934 (2020)

[32] J Dehning, J Zierenberg, FP Spitzner et. al., "Inferring change points in the spread of COVID-19 reveals the effectiveness of interventions," Science 369 (6500), eabb9789 (2020)

[33] K Prem, Y Liu, TW Russell et. al., "The Effect of control strategies to reduce social mixing on outcomes of the COVID-19 epidemic in Wuhan, China: a modelling study," The Lancet Public Health 5 (5) e261-e270 (2020)

[34] B Shayak and MM Sharma, "A New Approach to the Dynamic Modeling of an Infectious Disease," to be published in Mathematical Modelling of Natural Phenomena (2021). Available at https://www.mmnpjournal.org/component/article?access=doi\&doi=10.1051/mmnp/2021026

[35] NC Grassly, E Pons-Salort, EPK Parker et. al., "Comparison of molecular testing strategies for COVID-19 control: a mathematical modelling study," The Lancet Infectious Diseases 20 (12), 1381-1389 (2020)

[36] J Hellewell, S Abbott, A Gimma et. al., "Feasibility of controlling COVID-19 outbreaks by isolation of cases and contacts," The Lancet Global Health 8 (4), e488-e496 (2020)

[37] S Thurner, P Klimek and R Hanel, "A network-based explanation of why most COVID-19 infection curves are linear," Proceedings of the National Academy of Sciences USA (PNAS) 117 (37), 22684-22689 (2020)

[38] AV Tkachenko, S Maslov, T Wang et. al., "Stochastic social behaviour coupled to COVID-19 dynamics leads to waves, plateaus and an endemic state," MedRxiv Article (2021). Available at

https://www.medrxiv.org/content/10.1101/2021.01.28.21250701v3

[39] BF Nielsen, L Simonsen and K Sneppen, "COVID-19 superspreading suggests mitigation by social network modulation," Physical Review Letters 126 (11), 118301 (2021)

[40] S Manrubia and DH Zanette, "Individual risk aversion responses tune epidemics to critical transmissibility $(R=1), "$ Arxiv Article (2021). Available at https://arxiv.org/abs/2105.10572

[41] ICAO, "Aircraft Accident and Incident Investigation, Annex 13," (2001). Available at

https://www.emsa.europa.eu/retro/Docs/marine casualties/annex 13.pdf\%0Ahttp://www.emsa.europa.eu/retro/Docs/ marine casualties/annex 13.pdf

[42] https://en.wikipedia.org/wiki/List of busiest passenger air routes

[43] SA Kemp, DA Collier, RP Datir et. al., "SARS-CoV-2 evolution during treatment of chronic infection," Nature 592 (7853), 277-282 (2021)

[44] S Weigang, J Fuchs, G Zimmer, D Schnepf and L Kern, "With host evolution of SARS-CoV-2 in an immunosuppressed COVID-19 patient : a source of immune escape variants," MedRxiv Article (2021). Available at https://www.medrxiv.org/content/10.1101/2021.04.30.21256244v1

[45] F Karim, MYS Moosa, BI Gosnell et. al., "Persistent SARS-CoV-2 infection and intra-host evolution in association with advanced HIV infection," MedRxiv Article (2021). Available at 
https://www.medrxiv.org/content/10.1101/2021.06.03.21258228v1

[46] B Shayak, MM Sharma and AK Mishra, "COVID-19 Spreading Dynamics in an Age-Structured Population with Selective Relaxation of Restrictions for Vaccinated Individuals : a Mathematical Modeling Study," MedRxiv Article (2021). Available at https://www.medrxiv.org/content/10.1101/2021.02.22.21252241v1

[47] PR Haunschild and BN Sullivan, "Learning from Complexity: Effects of Prior Accidents and Incidents on Airlines' Learning," Administrative Science Quarterly 47 (4), 609-643 (2002) 